\title{
Outage Minimization with Limited Feedback for the Fading
}

\author{
Relay Channel \\ Nasir Ahmed, Mohammad Ali Khojastepour, Ashutosh Sabharwal and \\ Behnaam Aazhang \\ Department of Electrical and Computer Engineering \\ Rice University \\ email: \{nasir,amir,ashu,aaz\}@ rice.edu
}

\begin{abstract}
In this work, we consider practical methods to approach the theoretical performance limits in the fading relay channel under different assumptions of transmitter channel knowledge. Specifically, we consider two degrees of transmitter channel knowledge: (i) perfect feedback is available and power control is employed, and (ii) no channel state knowledge is available at the transmitters and only spatial power allocation is possible.

First, when perfect feedback is available, the optimal power control policy determines the ultimate limits of performance for constant rate transmission in the slow fading environment. However, in practice, perfect channel knowledge is not possible at the transmitters due to the finite capacity of the feedback links. We find practical methods to approach this performance limit through the use of power control with finite rate feedback. The finite rate feedback results are shown for the low complexity, full diversity amplify-and-forward(AF) protocol. Interestingly, we see that only a few feedback bits are needed to achieve most of the gains of the optimal perfect feedback power control algorithm.

Second, we consider the performance limit when the transmitters have no channel state knowledge, and derive the optimal spatial power allocation between the source and relay for a given sum power constraint for the AF protocol. For most practical cases of interest, equal power allocation between the source and relay is shown to be nearly optimal. Our work suggests that there is minimal power savings from using spatial power allocation at the transmitters. To obtain large performance improvements over
\end{abstract}


constant power transmission, it is imperative to have feedback for each realization of the channel state to allow for temporal power control.

\section{Index Terms}

Relay channel, Limited feedback, Block fading, Power control, Diversity methods.

\section{INTRODUCTION}

User cooperation is a powerful transmission technique that can improve the throughput over traditional point-to-point communications in wireless networks $[1,2]$. Exploiting channel knowledge at the transmitter for point-to-point communications leads to significant performance improvements [3-5]. However, even in the simplest form of cooperation, the relay channel, almost no attention has been paid to finding algorithms to make use of transmitter channel knowledge. In order to fully realize the benefits of cooperative transmission, feedback information must be exploited when it is available.

The objective of this work is to investigate methods to approach the performance limits in the fading relay channel under different assumptions of network channel state information at the transmitters (CSIT). The first performance limit considered is the one defined by the optimal power control policy when perfect network channel state information is available at the source and relay. However, in practice, having a perfect channel estimate at the transmitters is impractical, especially in network scenarios. Hence, we consider the effect of finite rate feedback links. We derive a power control policy based on the rate of the feedback link, and we show how it can be used to approach the perfect feedback power control limit. Second, when channel state information is unavailable to the transmitters, we find the optimal performance limit for a given protocol and provide a simple method to approach this limit.

To approach the performance of the optimal CSIT power control algorithm, we describe a power control procedure based on a limited feedback channel that is extendable to any number 
of feedback bits. Interestingly, we see that with just one or two bits of power control information, the finite rate feedback algorithm can overcome most of the performance gains that the optimal CSIT power control policy achieves over constant power transmission. Furthermore, we show a simple power control policy, where equal average power is given to each power control subregion. This practical policy allows for efficient computation of the power control regions, and is easily extendable to any rate of the feedback link. Our results are general and can be extended to many relay coding protocols. However, we show results based on the amplify-and-forward(AF) protocol [6], which is an attractive network code due to its simplicity and ability to achieve full diversity. ${ }^{1}$ For the AF technique, through an analysis of the outage probability, we are able to show that the use of a feedback bit doubles the diversity order over constant power transmission. The effect of the increased diversity order is a significant savings in power over constant power transmission for a target frame error rate. Such power savings are of particular importance in systems requiring energy efficiency, such as ad-hoc and sensor networks [9]. It is therefore imperative that next-generation network protocols utilize feedback to enable power control, as it will result in significant battery life improvements.

The second performance limit considered in this work occurs when no channel state knowledge is available to the transmitters. When no CSIT is available, then temporal power control is not possible. However, based on the statistics of the links in the network, the source and relay are able to determine the fraction of the total available power with which to transmit. For the AF protocol, we derive the optimal spatial power allocation between the source and relay. Interestingly, it is seen that for relays positioned close to the source, which is a scenario where relaying becomes feasible, equal power allocation between the source and relay is close to optimal. As a result, in the absence of CSIT, there is minimal power savings from using spatial power allocation at the

\footnotetext{
${ }^{1}$ In this work, we consider a more general definition of diversity. A diversity order of $d$ is obtained if for some constant $C$ and power $P$, the outage behaves as $\frac{C}{P^{d}}$. This is somewhat different from the traditional notion of diversity, which is obtained through the reception of independent paths (i.e. multiple transmit/receive antennas) of the data.
} 
transmitters. Our work suggests that to obtain large performance improvements over constant power transmission, it is imperative to have feedback for each realization of the channel state to allow for temporal power control.

The rest of the paper is organized as follows. Section II provides background regarding the protocols and models used throughout the paper. Section II.A discusses the general relay network and channel models. Section II.B describes the tools used for performance analysis. In Section II.C, we describe the AF protocol, which is the relay code used in this work. Section III investigates the outage performance of the relay protocol under the assumption that channel state information is available to the transmitters. Section IV considers power control with finite rate feedback. Section V looks at the case of no transmitter channel state information, and Section VI provides concluding remarks.

\section{PRELIMINARIES}

\section{A. Network Model}

Consider the relay network in Figure 1(a), with one relay node and one source-destination pair. The relay assists in the communication of data between the source and the destination, and it does not produce its own data. It is assumed that link $i$ in the network is attenuated by fading coefficient $h_{i}$, where $i \in\{0,1,2\}$. The magnitudes of these coefficients are assumed to follow a Rayleigh distribution. At both the source and relay, the received signal is corrupted by additive white Gaussian noise with zero mean and unit variance. The received signal at the relay is $y_{1}=h_{0} x_{1}+z_{1}$, where $x_{1}$ is the relay input and $z_{1}$ is the noise at the relay. At the destination, the received signal is $y=h_{1} x_{1}+h_{2} x_{2}+z$, where $x_{2}$ is the input signal at the relay, and $z$ is the noise at the destination.

In the sequel, we will denote $\gamma_{0}=\left|h_{0}\right|^{2}, \gamma_{1}=\left|h_{1}\right|^{2}$ and $\gamma_{2}=\left|h_{2}\right|^{2}$. The network channel state is defined by the 3-tuple $\underline{\gamma}=\left(\gamma_{0}, \gamma_{1}, \gamma_{2}\right)$, where $\gamma_{i}$ follows an exponential distribution with mean $\lambda_{i}, i \in\{0,1,2\}$. The parameter $\lambda_{i}$ captures the pathloss across link $i$ in the network, which is 
a function of the length of the link, and the pathloss exponent $\alpha$; typically, $\alpha$ lies in the range $(2,5)$.

To consider the effect of the relay nodes positioning, we use the model shown in Figure 1(b). We assume that the distance between the source and relay is one unit, and the relay is located in a line between the source and destination. The parameter $d$ represents the distance from the source to the relay, and $1-d$ is the distance from the relay to the destination. The mean value of the fading distribution for the source-relay link is consequently $\lambda_{0}=\frac{1}{d^{\alpha}}$ and for the relaydestination link we have $\lambda_{2}=\frac{1}{(1-d)^{\alpha}}$. To assess the effects of relay positioning on performance, for the remainder of this work, we consider the relay model of Figure 1(b).

\section{B. Performance Metric}

We consider a block fading model, where the fading coefficients $\gamma_{i}$ are constant over a block and are independent from one block to the next. A practical analysis tool for the block fading environment is the outage probability [13], which for large blocklengths, serves as a lower bound to the frame error rate, making it a practical tool for the analysis of coded systems. Outage probability is the probability that the instantaneous achievable rate of the channel is less than the transmission rate,

$$
P_{\text {out }}(R, \underline{\gamma})=\operatorname{Prob}\left[R>R_{\text {gen }}\left(\underline{\gamma}, P_{s}(\underline{\gamma}), P_{r}(\underline{\gamma})\right)\right]
$$

where $R_{g e n}$ is the instantaneous achievable rate of the transmission protocol used. In $(1), P_{s}(\underline{\gamma})$ is the transmit power of the source, $P_{r}(\underline{\gamma})$ is the transmit power of the relay and $R$ is the attempted rate of transmission. Note that in (1) the source and relay powers have been written as functions of the instantaneous network channel state $\underline{\gamma}$ to show that power control is possible when information regarding the network channel state is available to the transmitters. 


\section{Relaying Protocol}

Many network coding options are available, depending on the complexity and physical limitations of the relay node. An example of a physical limitation which can be used to classify coding protocols is the problem of 'cheap' relay nodes, introduced in [14], where transmission and reception simultaneously in the same frequency band is not possible. In this case, a practical transmission protocol is the amplify-and-forward (AF) technique, developed in [6]. This is a computationally efficient protocol since the operation at the relay is simply scaling and forwarding. Additionally, the source and relay transmissions are orthogonal, which eliminates any potential interference. Given a source with average power $P_{s}(\underline{\gamma})$ and a relay with average power $P_{r}(\underline{\gamma})$, the achievable rate of the AF transmission protocol is [6]

$$
R_{A F}\left(\underline{\gamma}, P_{s}(\underline{\gamma}), P_{r}(\underline{\gamma})\right)=\frac{1}{2} \log \left(1+2 \gamma_{1} P_{s}(\underline{\gamma})+\frac{4 \gamma_{2} P_{s}(\underline{\gamma}) \gamma_{0} P_{r}(\underline{\gamma})}{1+2 P_{s}(\underline{\gamma}) \gamma_{0}+2 P_{r}(\underline{\gamma}) \gamma_{2}}\right)
$$

Note that in (2), since each transmitter sends data for half the time slot, the source uses power $2 P_{s}(\underline{\gamma})$ and the relay uses power $2 P_{r}(\underline{\gamma})$ to guarantee an average power of $P_{s}(\underline{\gamma})+P_{r}(\underline{\gamma})$ per time slot. Note that calculating the outage probability of AF requires replacing $R_{g e n}(\cdot)$ in (1) with $R_{A F}(\cdot)$. Despite its simplicity, amplify-and-forward has been shown to achieve full diversity in a system with one relay node [6]. For this reason, the amplify-and-forward protocol is the relaying protocol studied in this work.

\section{Optimal Power Control with Perfect CSIT}

When the network channel state is available at the source and relay, outage minimization with power control can provide significant savings in power, as will be seen next. Given a network channel state of $\underline{\gamma}$ that is perfectly measured at the destination, the source and relay are instructed to transmit with powers $P_{s}(\underline{\gamma})$ and $P_{r}(\underline{\gamma})$, respectively. Assuming an arbitrary relay protocol with 
an achievable rate of $R_{g e n}\left(\underline{\gamma}, P_{s}(\underline{\gamma}), P_{r}(\underline{\gamma})\right)$, the outage probability becomes

$$
\begin{aligned}
P_{\text {out }}(R, \underline{\gamma})= & \operatorname{Prob}\left(R_{\text {gen }}\left(\underline{\gamma}, P_{s}(\underline{\gamma}), P_{r}(\underline{\gamma})\right)<R\right)= \\
& E_{\underline{\gamma}}\left[\mathcal{I}_{F}\left\{R_{g e n}\left(\underline{\gamma}, P_{s}(\underline{\gamma}), P_{r}(\underline{\gamma})\right)<R\right\}\right],
\end{aligned}
$$

where $\mathcal{I}_{F}(\cdot)$ is the indicator function. When outage minimization is performed with respect to an average long term sum power constraint, substantial reductions in outage are possible. The power constraint can be expressed as

$$
E_{\underline{\gamma}}\left[P_{s}(\underline{\gamma})+P_{r}(\underline{\gamma})\right] \leq 2 P_{a v g}
$$

Note that using the sum power constraint in (4) leads to an improved outage performance compared to the case of individual source and relay power constraints. However, the optimal power control policy found by using the constraint in (4) will serve as a lower bound on the outage probability of any finite rate feedback power control algorithm. The following proposition outlines the optimal power control strategy for the relay channel.

Proposition 1 ([11]): The optimal power allocation that minimizes the outage probability for a relaying protocol with achievable rate $R_{g e n}\left(\underline{\gamma}, P_{s}(\underline{\gamma}), P_{r}(\underline{\gamma})\right)$ under a long term sum power constraint is

$$
P_{L T}(\underline{\gamma})= \begin{cases}P_{s t}^{*}(\underline{\gamma}), & \text { with probability } 1, \text { if } P_{s t}^{*}(\underline{\gamma})<s^{*} \\ P_{s t}^{*}(\underline{\gamma}), & \text { with probability } w_{0}, \text { if } P_{s t}^{*}(\underline{\gamma})=s^{*} \\ 0, & \text { with probability } 1-w_{0}, \text { if } P_{s t}^{*}(\underline{\gamma})=s^{*} \\ 0, & \text { with probability } 1, \text { if } P_{s t}^{*}(\underline{\gamma})>s^{*} .\end{cases}
$$

The power level $P_{L T}(\underline{\gamma})$ is the sum of the instantaneous source and relay powers. Here $P_{s t}^{*}$ is the solution to $T\left(\underline{\gamma}, P_{s t}(\underline{\gamma})\right)=R$, and $P_{s t}$ is the short term sum power allocation which guarantees zero outage when transmitting at a rate $R$. Additionally, $w_{0} \in(0,1)$, and

$$
T\left(\underline{\gamma}, P_{s t}(\underline{\gamma})\right)=\max _{P_{s}(\underline{\gamma}), P_{r}(\underline{\gamma})}\left\{R_{g e n}\left(\underline{\gamma}, P_{s}(\underline{\gamma}), P_{r}(\underline{\gamma})\right): P_{s}(\underline{\gamma})+P_{r}(\underline{\gamma}) \leq 2 P_{s t}(\underline{\gamma})\right\}
$$

where $P_{s}(\underline{\gamma})$ is the instantaneous source power and $P_{r}(\underline{\gamma})$ is the instantaneous relay power. Furthermore, $s^{*}$ is chosen such that the average power constraint is satisfied. 
To summarize Proposition 1, minimizing the outage probability and satisfying the average sum power constraint involves first solving a short term power allocation problem that completely inverts the effects of the channel, and this is followed by finding a cutoff value for the sum power that guarantees the average power constraint. The power allocation procedure for the various relay protocols is similar, except the solution of $T\left(\underline{\gamma}, P_{s t}(\underline{\gamma})\right)$ varies depending on the form of the achievable rate.

In Figure 2, the outage probability is shown for the optimal power control policy between the source and relay. The relay is assumed to be at a distance of $d=0.5$, which leads to good sourcerelay and relay-destination links. The channel gains $\left|h_{i}\right|, i \in 0,1,2$ follow a Rayleigh distribution, and are independent of each other. The pathloss exponent is $\alpha=3$. At an outage probability of $10^{-2}$, we achieve more than $10 \mathrm{~dB}$ savings in power over constant power transmission through optimal power allocation. Clearly, this result motivates the need for feedback in relay networks. In the next section, we show how through a limited feedback link, we can redeem most of the gains that the algorithm in Proposition 1 achieves over constant power transmission.

\section{Power Control with Finite Rate Feedback}

In this section, we derive a power control algorithm for the relay channel that uses limited feedback. First, we outline the general procedure, and then we present a low complexity suboptimal solution. The low complexity solution has the property that it can be easily extended to an arbitrary number of feedback bits. For the case of one feedback bit, an approximation to the outage probability is developed, and the diversity gain for the AF protocol is shown to double over constant power transmission.

\section{A. General Procedure}

Consider the destination, which has a perfect estimate of the network channel state $\underline{\gamma}$. Given $M$ bits of feedback, the space defined by all possible sets of $\underline{\gamma}$ is quantized into $L=2^{M}$ 
regions. For the network channel state $\underline{\gamma}$, the region is a volume in the space defined by all positive $\left(\gamma_{0}, \gamma_{1}, \gamma_{2}\right)$. The destination, upon measuring the channel state, selects a power-tuple $\mathbf{P}_{\mathbf{q}}=\left(P_{s, q}, P_{r, q}\right)$, from a power control codebook $\mathcal{C}$, of size $L$, where $q \in\{1,2, \ldots, L\}$. The index $q$ to the selected power-tuple is transmitted to both the source and relay through a noiseless feedback link. It is assumed that both the source and relay have copies of $\mathcal{C}$. Upon reception of the index $q$, the source transmits with power $P_{s, q}$ and the relay with power $P_{r, q}$.

The elements of $\mathcal{C}$ are chosen to maintain the average power constraints of both the source and relay. We consider the case where both the source and relay have individual average power constraints. The power control policy described by Proposition 1 involves outage minimization with a sum power constraint, and serves as a lower bound to the outage for the case of individual power constraints on the source and relay. As a result, even as $L \rightarrow \infty$, the power control policy of Proposition 1 will provide a lower bound on the outage probability of the developed power control algorithm.

Consider the power control function $S: \mathbb{R}_{+}^{3} \rightarrow \mathbb{R}_{+}^{2}$, which maps the current channel state $\underline{\gamma} \in \mathbb{R}_{+}^{3}$ to a codebook element $\mathbf{P}_{\mathbf{q}} \in \mathbb{R}_{+}^{2}$. To satisfy the average power constraint, $E_{\underline{\gamma}}[S(\underline{\gamma})] \leq$ $\left(P_{s}, P_{r}\right)$ must hold on a per element basis. The objective of the power control algorithm is to find a $S(\underline{\gamma})$ that minimizes the outage probability while meeting the power constraint. In general, the elements of $\mathbf{P}_{\mathbf{q}}$ can differ, chosen to meet individual power constraints of the source and relay. To simplify the analysis, we impose one of two possible restrictions on $P_{r, q}$. The first restriction is where the relay transmits with a constant power $P_{r}$ in each time slot. This leads to a power-tuple of $\mathbf{P}_{\mathbf{q}}=\left(P_{s, q}, P_{r}\right)$. The second restriction is where the relay takes a similar action as the source, depending on its power constraint. That is, if $P_{r}=\eta P_{s}$, then we impose a constraint on power-tuple $q$ as $\mathbf{P}_{\mathbf{q}}=\left(P_{s, q}, \eta P_{s, q}\right)$. We will show later that the second form of the power-tuple allows for an increase in performance over using a constant relay power. The results presented next are applicable to both scenarios. 
Given $M$ bits of feedback, the space defined by all $\left(\gamma_{0}, \gamma_{1}, \gamma_{2}\right)$ will be divided into $L=$ $2^{M}$ subregions $\mathcal{R}_{q}, q \in\{1,2, \ldots, L\}$. If the instantaneous value of $\underline{\gamma}$ falls into region $\mathcal{R}_{q}$, the destination indicates to the source and relay to use power-tuple $\mathbf{P}_{\mathbf{q}}$. The power levels $\left(P_{s, q}, P_{r, q}\right)$, $q \in\{1,2, \ldots, L\}$ are chosen to satisfy the long term power constraint, i.e.,

$$
\left(P_{s}, P_{r}\right)=\left(\sum_{q=1}^{L} P_{s, q} \int_{\mathcal{R}_{q}} f(\underline{\gamma}) d \underline{\gamma}, \sum_{q=1}^{L} P_{r, q} \int_{\mathcal{R}_{q}} f(\underline{\gamma}) d \underline{\gamma}\right),
$$

where $f(\underline{\gamma})$ is the joint probability distribution of the network channel state $\underline{\gamma}$.

In Figure 3(a), for the amplify-and-forward protocol and for a given $\gamma_{0}$, the power control regions $\mathcal{R}_{q}$ are shown. Although we have shown the space of $\left(\gamma_{1}, \gamma_{2}\right)$ for a given $\gamma_{0}$, changing $\gamma_{0}$ changes the position of $\mu_{q}$. The power control regions are in fact volumes in the space defined by $\underline{\gamma}$, where for any particular $\gamma_{0}$, a cross-section of the 3 -D space is similar to that shown in Figure 3(a).

One key feature of the power control regions is that in region $\mathcal{R}_{q}, q \geq 2$, the assigned power $\mathbf{P}_{\mathbf{q}}$ is the minimum required to guarantee zero outage for any point in the region. This is a fundamental property of all optimal finite rate feedback power control algorithms [4]. With this property in mind, assuming a relaying protocol with achievable rate $R_{\text {gen }}$ and transmitting at a constant rate $R$, power level $P_{s, q}$ is the solution to

$$
\mathcal{R}_{g e n}\left(\underline{\gamma}, P_{s, q}, x\right)=R
$$

Note that in (8), $x=P_{r}$ when the relay power is always constant, and $x=\eta P_{s, q}$ when the relay also adapts its power.

From Figure 3(a) observe that for a fixed $\gamma_{0}$, the boundary between $\mathcal{R}_{q}$ and $\mathcal{R}_{q+1}$ is separated by a curve $G\left(\gamma_{0}, \gamma_{1}, \mathbf{P}_{\mathbf{q}+\mathbf{1}}\right)$. This curve is found by solving for $\gamma_{2}$ in (8). Any $\left(\gamma_{1}, \gamma_{2}\right)$ along this curve requires exactly powers $\mathbf{P}_{\mathbf{q}+\mathbf{1}}$ for zero outage, while any other points in $\mathcal{R}_{q+1}$ require instantaneous source and relay powers less than $P_{s, q}$ and $P_{r, q}$, respectively, for zero outage. We state this formally in the following theorem. 
Theorem 1: For the amplify-and-forward protocol, any points lying below the curve $G\left(\gamma_{0}, \gamma_{1}, \mathbf{P}_{\mathbf{q}}\right)$ require source and relay powers greater than $P_{s, q}$ and $P_{r, q}$, respectively, to guarantee zero outage. Furthermore, any points lying above this curve require source and relay powers less than $P_{s, q}$ and $P_{r, q}$, respectively, to guarantee zero outage.

Proof: See Appendix I.

As a result of Theorem 1, the entire region $\mathcal{R}_{q+1}$ has no outages. This property holds for all $\mathcal{R}_{q}, q \in\{2, \ldots, L\}$. Based on the power constraint, however, a portion of $\mathcal{R}_{1}$ would be in outage. Therefore, calculating the outage probability reduces to an analysis of region $\mathcal{R}_{1}$.

Region $\mathcal{R}_{1}$ uses power-tuple $\mathbf{P}_{1}$ corresponding to a source power of $P_{s, 1}$ and a relay power of $P_{r, 1}$. The outage probability is the probability that the source power required to invert the channel is greater than $P_{s, 1}$. Note that our analysis stems from the properties of the source power, as the relay power is either constant or a scaled version of the source power. Defining $P^{*}$ as the minimum power to guarantee zero outage for network channel state $\underline{\gamma}$, then $P^{*}$ can be written as the solution of

$$
R_{g e n}\left(\underline{\gamma}, P^{*}, x\right)=R
$$

where $x=P_{r}$ when the relay transmits with constant power, or else $x=\eta P^{*}$ when the relay also adapts its power. With the solution to $P^{*}$ in hand, the outage probability can be expressed as

$$
\Pi_{\text {out }}=\int_{\underline{\gamma}: P^{*} \geq P_{s, 1}} f(\underline{\gamma}) d \underline{\gamma} .
$$

Different relaying protocols will have different solutions of $G\left(\gamma_{0}, \gamma_{1}, \mathbf{P}_{\mathbf{q}}\right)$. Considering the amplify-and-forward protocol, solving for $\gamma_{2}$ leads to the following

$$
G_{A F}\left(\gamma_{0}, \gamma_{1}, \mathbf{P}_{\mathbf{q}}\right)=\frac{K\left(1+P_{s, q} \gamma_{0}\right)-P_{s, q} \gamma_{1}\left(1+P_{s, q} \gamma_{0}\right)}{P_{r, q}\left(-K+P_{s, q} \gamma_{1}+P_{s, q} \gamma_{0}\right)}
$$

where $K=e^{2 R}-1$. It can be easily verified that $\mu_{q}=K / P_{s, q}-\gamma_{0}$ for $q \in\{2, \ldots, L\}$, and $\pi_{q}=K / P_{s, q}$ for $q \in\{1, \ldots, L\}$, where $\pi_{q}$ and $\mu_{q}$ are defined in Figure 3(a). The power control 
regions for variable $\gamma_{0}$ can be visualized by considering the effect of $\gamma_{0}$ on $\mu_{q}$ and also in the form of $G_{A F}(\cdot)$.

\section{B. Suboptimal Power Control Method}

In general, solving the regions $\mathcal{R}_{q}$ and the associated power levels is computationally complex. However, for a more efficient approach, we consider a method similar to [19], where equal total power is allocated to each subregion [19]. For the case of multiple antenna systems with finite rate feedback, this technique was shown to be a good solution and close to optimal for large powers and for increasing bits of feedback. The power of this method is that, instead of jointly solving for the power control levels, they can be found in a successive fashion, which makes this algorithm amenable to a large number of feedback bits. The procedure is described next.

First, the power levels $\left(P_{s, L}, P_{r, L}\right)$ are solved by noting that

$$
\frac{P_{s}}{L}=P_{s, L} \int_{\mathcal{R}_{L}} f(\underline{\gamma}) d \underline{\gamma} .
$$

The solution determines the power levels $\left(P_{s, L}, P_{r, L}\right)$ and the region boundary $G\left(\gamma_{0}, \gamma_{1}, \mathbf{P}_{\mathbf{L}}\right)$. Once region $L$ has been solved, then region $L-1$ can be found. This process is continued until power level $\mathbf{P}_{\mathbf{1}}$ has been found. Solving power level $\mathbf{P}_{\mathbf{q}}$ requires knowledge of $\mathbf{P}_{\mathbf{q}+\mathbf{1}}$, and by the simplifying assumption that $\frac{P_{s}}{L}=P_{s, q} \int_{\mathcal{R}_{q}} f(\underline{\gamma}) d \underline{\gamma}$. Note that we have used the total power in each region as $P_{s} / L$, since the power level for each region $\mathcal{R}_{q}$ corresponds to the transmit power of the source, and the relay can either transmit with a constant $P_{r}$ or a variable power related to the source power. In either case, the relay's action is reflected in the algorithm by the form of $G\left(\gamma_{0}, \gamma_{1}, \mathbf{P}_{\mathbf{q}}\right)$ and hence in the solution of regions $\mathcal{R}_{q}$. In Section IV-D, we will see how using this suboptimal technique with just a few power levels leads to tremendous savings in power at a target outage probability over constant power transmission. 


\section{Lower Bound on Diversity Order}

It was seen in [6] that the amplify-and-forward protocol transmitting at constant power has a diversity order of two compared to a first order diversity for the single antenna direct transmission system. We next show that for the case of one bit of feedback, the diversity gain doubles from two to four for the AF protocol.

To show the behavior of 1-bit of feedback for the amplify-and-forward protocol, we first consider the effect of the source-relay fading value, $\gamma_{0}$. It should be noted that even in the case of a Gaussian source-relay link with a fixed $\gamma_{0}$, amplify-and-forward still exhibits a second order diversity. This can be shown rigorously by analyzing the asymptotic behavior of the exponential distribution.

Theorem 2: For the amplify-and-forward protocol with a fixed $\gamma_{0}$, and random $\gamma_{1}$ and $\gamma_{2}$, transmitting at a constant power leads to a second order diversity.

Proof: See Appendix II.

Aside from Theorem 2, the fact that a fixed value of $\gamma_{0}$ does not effect the diversity can also be understood by observing that the destination node still sees two independent copies of the information, through the random source-destination and the relay-destination links. With this in mind, we state the following theorem.

Theorem 3: For the amplify-and-forward protocol, as $P_{r}=P_{a v g}$ increases, the optimal one bit network power control offers at least a diversity order of four.

Proof: Assume that the source-relay link is Gaussian, with a fixed source-relay link gain of $\gamma_{0}$. As was proven in Theorem 2, this assumption does not affect the diversity order analysis. Additionally, it is assumed that the relay simply transmits with power $P_{r}$ in each time slot, as we are seeking a lower bound for diversity order. The analysis for outage probability that follows assumes large values of SNR. Under such a scenario, the hyperbola shown in Figure 3(b) intersects the $\gamma_{2}$ axis. To compute a lower bound to the outage probability under such a scenario, 
we approximate the hyperbola as a triangle, as seen in Figure 4. The concavity of the hyperbola guarantees that the approximate outage analysis will be a lower bound, since $P_{s, 1} \geq P_{s, 2}$ for large power constraints. Looking in $R_{1}$, a line defined as $C_{l}\left(\gamma_{0}, \gamma_{1}, \mathbf{P}_{\mathbf{1}}\right)=\delta_{\text {out }}-\gamma_{\mathbf{1}} \delta_{\text {out }} / \gamma_{\text {out }}$ defines the outage region. Also, in this figure the line $C_{l}\left(\gamma_{0}, \gamma_{1}, \mathbf{P}_{\mathbf{2}}\right)=\delta_{\mathbf{B}}-\gamma_{\mathbf{1}} \delta_{\mathbf{B}} / \gamma_{\mathbf{B}}$ is the boundary between $R_{1}$ and $R_{2}$. Note that $\gamma_{o u t}=K / P_{s, 1}$ and points below this curve are assumed to be in outage. Also, $\delta_{\text {out }}$ is found by setting $\gamma_{1}=0$ in $G_{A F}\left(\gamma_{0}, \gamma_{1},\left(P_{s, 1}, P_{r}\right)\right)$, and $\delta_{B}$ is found by solving for $\gamma_{2}$ in $G_{A F}\left(\gamma_{0}, 0,\left(P_{s, 2}, P_{r}\right)\right)$. The outage probability can be written as

$$
\Pi_{\text {out }}=\int_{\gamma_{1}=0}^{\gamma_{\text {out }}} \int_{\gamma_{2}=0}^{C_{l}\left(\gamma_{0}, 0, P_{s, 1}\right)} f_{\gamma_{1}, \gamma_{2}}\left(\gamma_{1}, \gamma_{2}\right) d \gamma_{1} d \gamma_{2}
$$

Denoting the probability that the network state $\left(\gamma_{1}, \gamma_{2}\right)$ is in region $R_{2}$ as $\Delta_{2}$, we can then write

$$
\Delta_{2}=\frac{1}{\lambda_{1} \lambda_{2}} \int_{\gamma_{1}=\gamma_{B}}^{\infty} e^{-\frac{\gamma_{1}}{\lambda_{1}}} \int_{\gamma_{2}=0}^{\infty} e^{-\frac{\gamma_{2}}{\lambda_{2}}} d \gamma_{2} \gamma_{1}+\frac{1}{\lambda_{1} \lambda_{2}} \int_{\gamma_{1}=0}^{\gamma_{B}} e^{-\frac{\gamma_{1}}{\lambda_{1}}} \int_{\gamma_{2}=C_{l}\left(\gamma_{0}, \gamma_{1}, P_{s, 2}\right)}^{\infty} e^{-\frac{\gamma_{2}}{\lambda_{2}}} d \gamma_{2} d \gamma_{1} .
$$

Using the second order Taylor approximation to the exponential function, $e^{-x} \approx 1-x+\frac{x^{2}}{2}$, it can be shown that $\Delta_{2} \approx 1-\frac{\delta_{B} \gamma_{B}}{2 \lambda_{1} \lambda_{2}}$. Using similar arguments, it can be shown that $\Pi_{\text {out }}=\frac{\delta_{\text {out }} \gamma_{\text {out }}}{2 \lambda_{1} \lambda_{2}}$, where $\gamma_{o u t}=K / P_{s, 1}$. Therefore, we have the following approximation for the outage probability for large $P_{s, 1}$

$$
\Pi_{\text {out }}=\frac{\delta_{\text {out }} \gamma_{\text {out }}}{2 \lambda_{1} \lambda_{2}}=\frac{K^{2}\left(1+P_{s, 1} \gamma_{0}\right)}{2 P_{r} P_{s, 1}\left(P_{s, 1} \gamma_{0}-K\right) \lambda_{1} \lambda_{2}} \approx \frac{K^{2}}{2 P_{r} P_{s, 1} \lambda_{1} \lambda_{2}} .
$$

To complete the analysis, we need to find $P_{s, 1}$ as a function of $P_{a v g}$. Using the power constraint on region $R_{1}$, we can show that $P_{s, 1}\left(1-\Delta_{2}\right)=P_{a v g} / 2$, which leads to $P_{s, 1}=\frac{P_{a v g} P_{r} P_{s, 2} \lambda_{1} \lambda_{2}\left(P_{s, 2} \gamma_{0}-K\right)}{K^{2}\left(P_{s, 2} \gamma_{0}+1\right)}$. With this in mind, the outage probability is now rewritten as a function of $P_{s, 2}$ as

$$
\Pi_{\text {out }} \approx \frac{K^{4}\left(P_{s, 2} \gamma_{0}+1\right)}{2 \lambda_{1}^{2} \lambda_{2}^{2} P_{r}^{2} P_{\text {avg }} P_{s, 2}\left(P_{s, 2} \gamma_{0}-K\right)}
$$

Clearly, (15) has a fourth order decay with respect to power $P_{a v g}$ as long as $P_{s, 2}$ is a linear function of $\mathrm{P}$ and when $P_{r}=P_{a v g}$. Next, $P_{s, 2}$ is found as a function of $P$. Using the fact that we are considering an algorithm where each power control region has the same total power, i.e., 
$P_{s, 2} \cdot \Delta_{2}=\frac{P_{a v g}}{2}, P_{s, 2}$ is the solution to the following

$$
P_{s, 2} \approx \frac{P_{a v g}}{4}+\frac{K}{2 \gamma_{0}}+\frac{\sqrt{\left(P_{a v g} / \gamma_{0}\right)^{2}+2 K^{2} /\left(\lambda_{1} \lambda_{2}\right)-4 P_{a v g} K / \gamma_{0}+4 K^{2} / \gamma_{0}^{2}}}{4} .
$$

As a result, since $P_{s, 2}$ depends on $P_{a v g}$, substitution into (15) leads to a fourth order decay of the outage probability as a function of the power.

From this result, it is clear that, with the use of just one feedback bit, the diversity order has doubled from two to four. A similar effect was seen in [19], for the case of direct transmission, where the decay in outage probability was proportional to the number of elements in the power control codebook. An additional point of interest is the effect of the mean values of the fading links $\lambda_{1}$ and $\lambda_{2}$. Increased values of these parameters lead to a decrease in the outage probability. However, the diversity order is still four. For the case of constant power transmission using the AF protocol, changes in $\lambda_{1}$ and $\lambda_{2}$ also do not effect the diversity order [6].

In [6], the authors explored the use of one feedback bit to improve system performance and proposed a technique known as incremental relaying. This relay protocol makes more efficient use of the available degrees of freedom by using a feedback bit to indicate the success/failure of the source transmission to the destination and only relaying when the source transmission leads to a decoding failure. This results in gains over traditional AF by increasing the rate for good source-relay conditions. However, it is shown in [6] that such a technique only provides a diversity order of 2 . Our work reveals that it is more efficient to use the feedback information for power control, which can lead to tremendous power savings over incremental relaying at the same transmission rate.

\section{Analysis and Discussion}

In Figure 2, power control with one bit of feedback for the AF protocol is shown. With just one bit of feedback, fourth order diversity is obtained, compared to a second order diversity for constant power transmission. At an outage probability of $10^{-2}$, there is approximately $5 \mathrm{~dB}$ of 
power savings with just one feedback bit. Furthermore, we observe that, at this same outage probability, one bit of feedback substantially reduces the gap to the optimal power control strategy. This motivates the need for future network protocols to allocate a few bits in feedback packets to allow for power control.

Recall that two possibilities were described for the action of the relay. First, the relay transmits with a constant power in each time slot. Second, the relay takes the same action as the source (when they have the same average power constraints). In Figure 2, for the case of 1-feedback bit, the gains of using a variable relay power are also shown. We see that there is a small gain from performing this type of power adaptation at the relay.

In Figure 5, for the amplify-and-forward protocol, the effect of increasing feedback bits is shown. Constant power transmission is compared to the proposed power control strategy with 2 power levels (1 bit feedback). Additionally, the gain from adding one more power control level is shown, and we see that, for small outage probabilities, much of the gap to the optimal power control strategy has been bridged. This suggests that only a few bits of feedback are necessary to extract large savings in power, and further increases in the feedback rate offer diminishing returns. Also shown in the figure is the performance of a direct transmission system using the same total power as AF and transmitting at the same rate. Clearly, direct transmission offers only a first order diversity, whereas AF has double this diversity, which translates into large power savings.

\section{Outage Minimization with No CSIT}

In the previous sections, the potential gains of using the optimal power control strategy were seen, and also the effects of limited feedback on outage minimization. We observed that only a few bits of feedback are needed to bridge much of the gap to the optimal CSIT power control algorithm. We next consider the case where the transmitters have no channel state information(CSIT) and thus cannot perform temporal power control. 
Even though the transmit powers for the source and relay are fixed, the outage probability can be minimized by determining the optimal fraction of the total power to be allocated to the source and relay. In each time slot, we have that $P_{s}+P_{r}=2 P_{\text {avg }}$. The objective is to find a $\kappa \in(0,1)$ such that the outage probability is minimized given that $P_{s}=2 P_{\text {avg }} \kappa$ and $P_{r}=2 P_{\text {avg }}(1-\kappa)$. In addition to the derivation of the optimal source-relay power ratio $\kappa^{*}$, we will see how the practical choice of using equal power at the source and relay performs close to optimal for many cases of interest. Next, we consider the performance of the amplify-and-forward protocol for the case of constant power transmission.

Consider the amplify-and-forward protocol and an optimal source-relay power ratio $\kappa^{*}$. The achievable rate is

$$
R_{A F}\left(\underline{\gamma}, P_{s}, P_{r}\right)=\frac{1}{2} \log \left(1+2 \gamma_{1} P_{a v g} \kappa^{*}+\frac{4 \gamma_{2} P_{a v g}^{2} \kappa^{*} \gamma_{0}\left(1-\kappa^{*}\right)}{1+2 P_{a v g} \kappa^{*} \gamma_{0}+2 P_{a v g}\left(1-\kappa^{*}\right) \gamma_{2}}\right) .
$$

We next characterize the outage probability for the amplify-and-forward protocol in the limit for large powers and for a given $\kappa$.

Lemma 1: As the average power $2 P_{\text {avg }}$ becomes large, the outage probability of the amplifyand-forward protocol can be approximated as

$$
P_{\text {out }} \approx\left\{\frac{e^{2 R}-1}{2 \sqrt{2} P_{\text {avg }}}\right\}^{2}\left[\frac{1}{\kappa \lambda_{1}} \cdot \frac{(1-\kappa) \lambda_{2}+\kappa \lambda_{0}}{\kappa(1-\kappa) \lambda_{0} \lambda_{2}}\right]
$$

where $\lambda_{i}, i \in\{0,1,2\}$ is the mean value of the fading for link $i$ in the relay network and $0 \leq \kappa \leq 1$ allocates power between the source and relay.

Proof: The proof is based on asymptotic analysis of the exponential distribution, which was described in [6]. The total network power is $2 P_{\text {avg }}$. Based on [6], it can be shown that

$$
\lim _{s \rightarrow \infty} s \cdot \operatorname{Prob}\left[s \kappa \gamma_{1}<t\right]=\frac{t}{\lambda_{1} \kappa}=f(t),
$$

and

$$
\lim _{s \rightarrow \infty} s \cdot \operatorname{Prob}\left[f\left(s \kappa \gamma_{0}, s(1-\kappa) \gamma_{2}\right)<t\right]=\frac{t}{\lambda_{0} \kappa}+\frac{t}{\lambda_{2}(1-\kappa)}=g(t),
$$


where $f(x, y)=\frac{x \cdot y}{x+y+1}$. In the above formulations, $s \kappa$ is the total power used by the source and $s(1-\kappa)$ is the power used by the relay. Using Theorem 1 from [7],

$$
\begin{gathered}
\lim _{s \rightarrow \infty} s^{2} \cdot \operatorname{Prob}\left[s \kappa \gamma_{1}+f\left(s \kappa \gamma_{0}, s(1-\kappa) \gamma_{2}\right)<t\right]= \\
\int_{0}^{t} g(t-x) \cdot f^{\prime}(x) d x= \\
\frac{t^{2}}{2 \kappa \lambda_{1}}\left[\frac{1}{\kappa \lambda_{0}}+\frac{1}{(1-\kappa) \lambda_{2}}\right] .
\end{gathered}
$$

Substituting $s^{2}=4 P_{\text {avg }}^{2}$ and $t=e^{2 R}-1$, the result follows.

We next investigate how the optimal source power $2 P_{a v g} \kappa^{*}$ is a function of the position of the relay. To do this, we consider again the scenario where the source and destination are one unit apart, and the relay is a distance $0 \leq d \leq 1$ from the source. Given a pathloss exponent $\alpha$, this leads to $\lambda_{1}=1, \lambda_{0}=\frac{1}{d^{\alpha}}$ and $\lambda_{2}=\frac{1}{(1-d)^{\alpha}}$. To find the optimal source-relay power ratio, it suffices to minimize the outage probability of (16) over all $\kappa$. Performing the minimization, the optimal value of $\kappa$ is

$$
\kappa^{*}=\frac{(1-d)^{\alpha}-4 d^{\alpha}+\sqrt{(1-d)^{2 \alpha}+8(1-d)^{\alpha} d^{\alpha}}}{4(1-d)^{\alpha}-4 d^{\alpha}} .
$$

An interesting property of the power ratio is that the solution is independent of the network power constraint $2 P_{a v g}$. Another interesting point is that the solution $\kappa^{*} \geq 0.5$, meaning that the relay should never transmit with more power than the source. In Figure 6, the savings in power by using the optimal power ratio of (17) are seen. By using the optimal ratio, up to $3 \mathrm{~dB}$ is saved over equal power allocation between the source and relay. However, we see that for small distances $(d<0.5)$, the gains of using the optimal ratio are minimal. As a result, when the source is close to the destination, using equal power for the source and relay is a good strategy.

\section{CONCLUSIONS}

In this work, we have analyzed power control methods to approach the fundamental limits in the fading relay channel for varying degrees of side information at the source and relay. When 
perfect side information is available at the transmitters, significant energy savings over constant power transmission can be obtained through optimal power control. However, we showed that only a few bits of feedback are sufficient to achieve most of the gains of the optimal CSIT power control policy. This result suggests the importance of designing protocols that incorporate feedback in future wireless networks, as even limited amounts of feedback will translate to significant increases in battery life for mobile nodes.

We also analyzed the case where no side information was available at the transmitters. Interestingly, transmitting with equal power at the source and relay is close to optimal, especially for relays positioned close to the source. This hints at the importance of the relay's contribution in improving system performance, as the power of the relay needs to be similar to the source's power in order to minimize the outage.

\section{APPENDIX I: PROOF OF THEOREM 1}

Note that $\mathcal{R}_{A F}\left(\underline{\gamma}, P_{s, q}, x\right)$ is a monotonically increasing function of $P_{s, q}$, for $x \in\left\{P_{r}, \eta P_{s, q}\right\}$.

This can be verified by confirming that $\frac{\partial \mathcal{R}_{A F}\left(\underline{\gamma}, P_{s, q}, x\right)}{P_{s, q}}>0$. Recall that $G(\cdot)$ is found by solving for $\gamma_{2}$ in $\mathcal{R}_{\text {gen }}\left(\underline{\gamma}, P_{s, q}, x\right)=R$. For any $\underline{\gamma}_{a}=\left(\gamma_{0}, \gamma_{1}, \gamma_{2}\right)$ lying on $G(\cdot)$, we will next show that for any $\underline{\gamma}_{b}=\left(\gamma_{0}-\epsilon_{0}, \gamma_{1}-\epsilon_{1}, \gamma_{2}-\epsilon_{2}\right), \mathcal{R}_{A F}\left(\underline{\gamma}_{b}, P_{s, q}, x\right)<\mathcal{R}_{A F}\left(\underline{\gamma}_{a}, P_{s, q}, x\right)$. As a result, since $\mathcal{R}_{A F}(\cdot)$ is monotonically increasing in the source power, to transmit at rate $R$ with new power $P_{s, q}^{b}$, an increase in source power $\left(P_{s, q}^{b}>P_{s, q}\right)$ is necessary to guarantee $\mathcal{R}_{A F}\left(\underline{\gamma}_{b}, P_{s, q}^{b}, x^{b}\right)=R$, where $x^{b} \in\left\{P_{r}, \eta P_{s, q}^{b}\right\}$.

For AF, consider $y(\underline{\gamma})=P_{s, q} \gamma_{1}+\frac{P_{s, q} \gamma_{0} x \gamma_{2}}{1+P_{s, q} \gamma_{0}+x \gamma_{2}}$. Let $y_{1}(\underline{\gamma})=1+P_{s, q} \gamma_{1}$, and $y_{2}(\underline{\gamma})=\frac{P_{s, q} \gamma_{0} x \gamma_{2}}{1+P_{s, q} \gamma_{0}+x \gamma_{2}}$. For $y_{1}(\underline{\gamma})$, clearly $1+P_{s, q} \gamma_{1}>1+P_{s, q}\left(\gamma_{1}-\epsilon_{1}\right)$. For $y_{2}(\underline{\gamma})$, it needs to be shown that

$$
\frac{P_{s, q} \gamma_{0} x\left(\gamma_{2}-\epsilon_{2}\right)}{1+P_{s, q}\left(\gamma_{0}-\epsilon_{0}\right)+x\left(\gamma_{2}-\epsilon_{2}\right)}<\frac{P_{s, q} \gamma_{0} x \gamma_{2}}{1+P_{s, q} \gamma_{0}+x \gamma_{2}} .
$$

After some manipulation, this can be rewritten as

$$
\left(1+P_{s, q}\right)\left(\frac{\gamma_{0}}{\epsilon_{0}}-1\right)+(1+x)\left(\frac{\gamma_{2}}{\epsilon_{2}}-1\right)>-1 .
$$


Since all fading elements are positive, then $\epsilon_{i} \leq \gamma_{i}$. As a result, (19) is always satisfied. We have then shown that $\frac{1}{2} \log \left(y\left(\underline{\gamma}-\left(\epsilon_{0}, \epsilon_{1}, \epsilon_{2}\right)\right)\right)<\frac{1}{2} \log (y(\underline{\gamma}))$ which corresponds to $\mathcal{R}_{A F}\left(\underline{\gamma}_{b}, P_{s, q}, x\right)<$ $\mathcal{R}_{A F}\left(\underline{\gamma}_{a}, P_{s, q}, x\right)$. Consequently, an increase in source power is required to guarantee zero outage for any channel state lying below the curve $G(\cdot)$.

Proving the second part of the theorem, that points lying above $G\left(\gamma_{0}, \gamma_{1}, \mathbf{P}_{\mathbf{q}}\right)$ requires a source power less than $P_{s, q}$ and a relay power less than $P_{r, q}$, is straightforward. By following similar steps as above, except now setting $\underline{\gamma}_{b}=\left(\gamma_{0}+\epsilon_{0}, \gamma_{1}+\epsilon_{1}, \gamma_{2}+\epsilon_{2}\right)$, the result follows.

\section{APPENDIX II: PROOF OF THEOREM 2}

Consider the function $g\left(P_{a v g}, \gamma_{0}, \gamma_{2}\right)=\frac{P_{a v g}^{2} \gamma_{0} \gamma_{2}}{1+P_{a v g} \gamma_{0}+P_{a v g} \gamma_{2}}$. We first will find $\operatorname{Pr}\left[g\left(P_{a v g}, \gamma_{0}, \gamma_{2}\right)<\right.$ $t]$. This can be re-written as $\operatorname{Pr}\left[1 / g\left(P_{a v g}, \gamma_{0}, \gamma_{2}\right)>1 / t\right]$. Next, note that

$$
\begin{array}{r}
\operatorname{Pr}\left[1 / g\left(P_{a v g}, \gamma_{0}, \gamma_{2}\right)>1 / t\right]=\operatorname{Pr}\left[\frac{1+P_{a v g} \gamma_{0}+P_{a v g} \gamma_{2}}{P_{a v g}^{2} \gamma_{0} \gamma_{2}}>\frac{1}{t}\right]= \\
\operatorname{Pr}\left[\gamma_{2}<\frac{t\left(1+P_{a v g} \gamma_{0}\right)}{P_{a v g}\left(P_{a v g} \gamma_{0}-t\right)}\right]= \\
1-\exp \left(-\frac{t\left(1+P_{a v g} \gamma_{0}\right)}{\lambda_{2} P_{a v g}\left(P_{a v g} \gamma_{0}-t\right)}\right) .
\end{array}
$$

Next, using the fact that $e^{-x}=1-x+x^{2} / 2+\ldots$, we can approximate $\lim _{P_{a v g} \rightarrow \infty}\left(P_{\text {avg }}\right.$. $\left.\operatorname{Pr}\left[g\left(P_{\text {avg }}, \gamma_{0}, \gamma_{2}\right)<t\right]\right)$ as

$$
\lim _{P_{a v g} \rightarrow \infty}\left(P_{a v g} \cdot \operatorname{Pr}\left[g\left(P_{a v g}, \gamma_{0}, \gamma_{2}\right)<t\right]\right)=\lim _{P_{a v g} \rightarrow \infty} \frac{P_{a v g} t\left(1+P_{a v g} \gamma_{0}\right)}{\lambda_{2} P_{a v g}\left(P_{a v g} \gamma_{0}-t\right)}=\frac{t P_{a v g} \gamma_{0}}{\lambda_{2}\left(P_{a v g} \gamma_{0}-t\right)}=\frac{t}{\lambda_{2}} .
$$

Next, consider $\operatorname{Pr}\left[P_{a v g} \gamma_{1}<t\right]$. It can be verified that

$$
\lim _{P_{a v g} \rightarrow \infty}\left(P_{a v g} \cdot \operatorname{Pr}\left[P_{a v g} \gamma_{1}<t\right]\right)=\lim _{P_{a v g} \rightarrow \infty}\left(P_{a v g} \cdot\left(1-e^{-\frac{t}{\operatorname{Pavg}_{1}}}\right)\right)=\frac{t}{\lambda_{1}}
$$

Let $a(t)=t / \lambda_{1}$, and $b(t)=t / \lambda_{2}$. Using Theorem 1 from [7],

$$
\lim _{P_{a v g} \rightarrow \infty} P_{a v g}^{2} \cdot \operatorname{Pr}\left(P_{a v g} \gamma_{1}+\frac{P_{a v g}^{2} \gamma_{0} \gamma_{2}}{1+P_{a v g} \gamma_{0}+P_{a v g} \gamma_{2}}<t\right)=\int_{0}^{t} a(t-x) b^{\prime}(x) d x=\frac{t^{2}}{2 \lambda_{1} \lambda_{2}}
$$

Using the fact that $t=e^{2 R}-1$, for large $P$, we have that

$$
\operatorname{Pr}\left(P_{a v g} \gamma_{1}+\frac{P_{a v g}^{2} \gamma_{0} \gamma_{2}}{1+P_{a v g} \gamma_{0}+P \gamma_{2}}<e^{2 R}-1\right) \approx \frac{\left(e^{2 R}-1\right)^{2}}{2 P_{a v g}^{2} \lambda_{1} \lambda_{2}}
$$


Clearly, there is a second order decay of the outage with respect to power for this case. As a result, a deterministic $\gamma_{0}$ does not affect the diversity order.

\section{REFERENCES}

[1] A. Sendonaris and E. Erkip and B. Aazhang, User Cooperation Diversity-Part I: System Description, IEEE Transactions on Communications, vol. 51, no. 11, 1927-1938,Nov. 2003.

[2] A. Sendonaris and E. Erkip and B. Aazhang, User Cooperation Diversity-Part II: Implementation Aspects and Performance Analysis, IEEE Transactions on Communications, vol. 51, no. 11, pp., 1939-1948, Nov. 2003.

[3] G. Caire, G. Taricco and E. Biglieri, "Optimum power control over fading channels," IEEE Trans. on Info. Theory., vol. 45, no. 5, pp., 1468-1489, July 1998.

[4] S. Bhashyam, A. Sabharwal and B. Aazhang, "Feedback Gain in Multiple Antenna Systems," IEEE Trans. on Comm., vol. 50, no. 5, pp., 795-798, May 2002.

[5] K. Mukkavilli, A. Sabharwal, E. Erkip and B. Aazhang, "On Beamforming with Finite Rate Feedback in Multiple-Antenna Systems," IEEE Trans. on Info. Theory, vol. 49, no. 10, pp., 2562-2579, Oct. 2003.

[6] N. Laneman, D. Tse and G. Wornell "Coooperative Diversity in Wireless Networks: Efficient Protocols and Outage Behavior," IEEE Trans. on Info. Theory., vol. 50, no. 12, Dec. 2004

[7] N. Laneman "Limiting Analysis of Outage Probabilities for Diversity Schemes in Fading Channels," in Proc. IEEE Global Commun. Conf. (GLOBECOM). Dec. 2003, San Franciso, CA..

[8] N. Laneman and G. Wornell "Distributed Space-Time Coded Protocols for Exploiting Cooperative Diversity in Wireless Networks," To appear in IEEE Transactions on Information Theory,

[9] Y. Xu, S. Bien, Y. Mori, J. Heidmann, D. Estrin, "Topology Control Protocols to Conserve Energy in Wireless Ad Hoc Networks," Under Submission to CENS Technical Report 0006, Jan. 2003.

[10] N. Ahmed, M. Khojastepour, A. Sabharwal, and B. Aazhang Power Control with Finite Rate Feedback for Cooperative Relay Networks, Intern. Symposium on Inform. Theory and its Applications (ISITA), Oct. 10-13, Parma, Italy, 2004.

[11] N. Ahmed, M. Khojastepour, and B. Aazhang Outage Minimization and Optimal Power Control for the Fading Relay Channel, IEEE Information Theory Workshop (ITW), Oct. 24-29, San Antonio, TX, 2004.

[12] M. Khojastepour, N. Ahmed, and B. Aazhang Performance Limits of the Fading Relay Channel - Part I: Ergodic Capacity, To be submitted to IEEE Trans. on Info. Theory,

[13] L. H. Ozarow, S. Shamai and A. D. Wyner, Information theoretic considerations for cellular mobile radio, IEEE Trans. on Vehicular Technology, vol. 43, num. 2, pp. 359-378, May 1994.

[14] M. A. Khojastepour, A. Sabharwal and B. Aazhang, "Lower Bounds on the Capacity of Gaussian Relay Channel," In Proc. $38^{\text {th }}$ Annual Conference on Information Sciences and Systems March 17-19, Princeton, NJ., 2004.

[15] M. A. Khojastepour, A. Sabharwal and B. Aazhang, "On the Capacity of 'Cheap' Relay Networks," In Proc. 37th Annual Conf. on Info. Sciences and Systems, March 12-14, Baltimore, MD., 2003.

[16] R. Nabar and H. Bolcskei and F. Kneubuhler "Fading Relay Channels: Performance limits and space-time signal design," IEEE Journ. on Selected Areas in Comm (JSAC), vol. 22, no. 6, pp. 1099-1109, Aug. 2004.

[17] T.M. Cover and A. E. Gamal "Capacity theorems for the relay channel," IEEE Trans. on Info. Theory, vol. 25, no. 5, pp., 572-584, Sept 1979.

[18] E. C. van der Meulen, "Three-terminal communication channels," Adv. Appl. Prob., vol. 3, 1971.

[19] A. Khoshnevis and A. Sabharwal, Performance of Quantized Power Control in Multiple Antenna Systems, In IEEE International Conference on Communications (ICC). June 20-24, Paris, France, 2004.

[20] G. Kramer and M. Gastpar and P. Gupta, "Capacity theorems for wireless relay channels," In Proc. 41st Annual Allerton Conf. on Commun., Control and Comp. Monticello, IL., Oct. 2004.

[21] A. Host-Madsen and J. Zhang, Capacity Bounds and Power Allocation for Wireless Relay Channel, Submitted to IEEE Trans. on Info. Theory, 


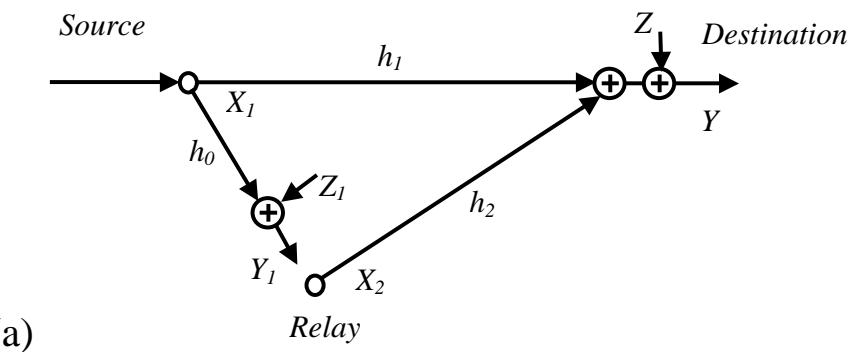

(b)

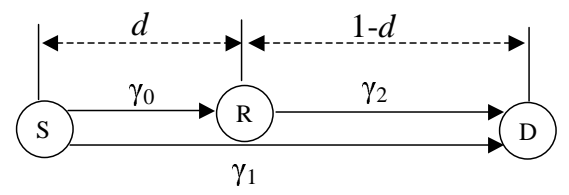

Fig. 1. (a) Layout of the relay network with 3 nodes. The source transmits to the destination, and the relay node assists in the communication process. Communication along the links are corrupted by pathloss along the links in the network and Gaussian noise at the receivers; (b) Layout of the relay network with the relay node located along a straight line from the source to the destination. Assuming the fading value is inversely proportional to the distance, then $E\left[\gamma_{0}\right]=1, E\left[\gamma_{1}\right]=\frac{1}{d^{\alpha}}$ and $E\left[\gamma_{2}\right]=\frac{1}{(1-d)^{\alpha}}$.

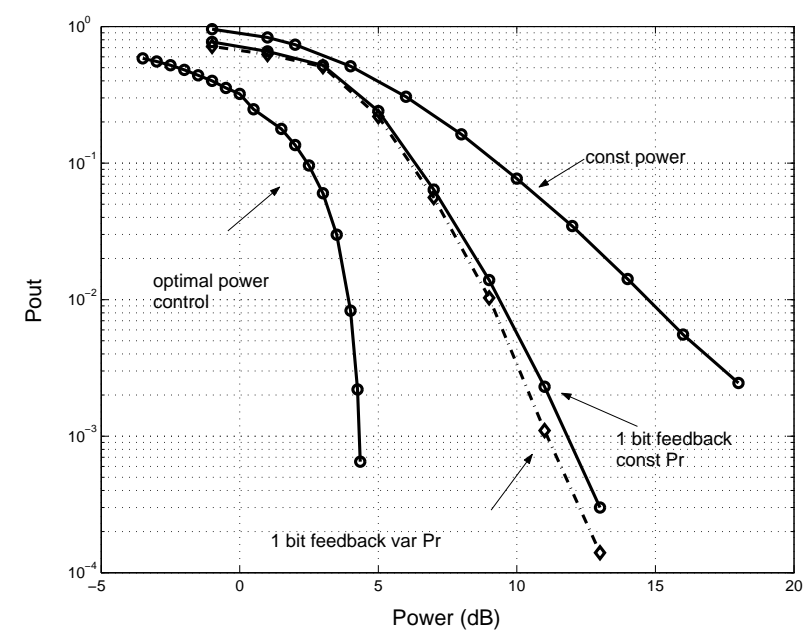

Fig. 2. Outage performance vs. total network power for the amplify-and-forward scheme, with $\mathrm{d}=0.5, \mathrm{R}=1 \mathrm{nats} / \mathrm{sec} / \mathrm{Hz}$ and $\alpha=3$. For the case of 1 feedback bit, the solid line indicates a constant $P_{r}$, and a dashed line indicates a variable $P_{r}$. The source and relay are given equal average power constraints.

(a)

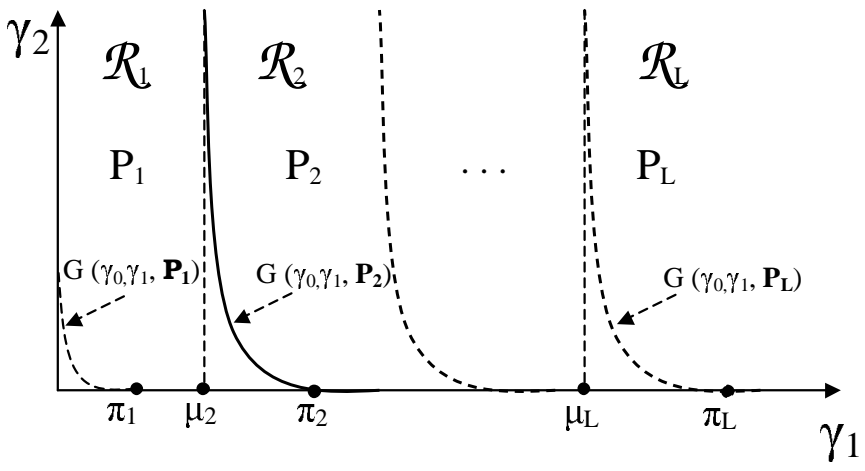

(b)

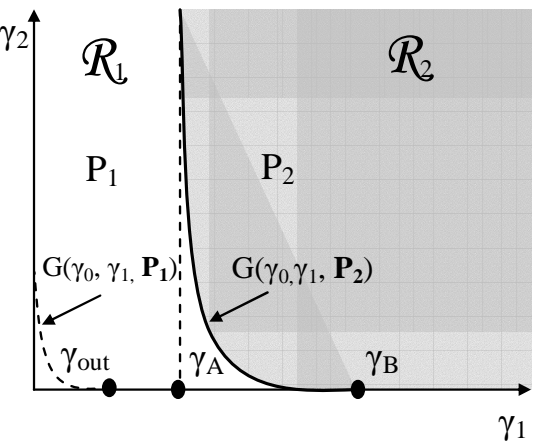

Fig. 3. (a) Structure of power control regions for a fixed $\gamma_{0}$. Using $\log _{2} L$ bits of feedback, the space of all $\left(\gamma_{1}, \gamma_{2}\right)$ is divided into $\mathrm{L}$ subregions. In region $\mathcal{R}_{i}, i \in\{1, \ldots, L\}$, power level $P_{i}$ is used; (b) Structure of power control regions for a fixed $\gamma_{0}$ and 2 subregions. The function $G\left(\gamma_{0}, \gamma_{1}, P_{1}\right)$ defines the outage region such that all points lying below this curve require more than power $P_{1}$ to guarantee zero outage. 


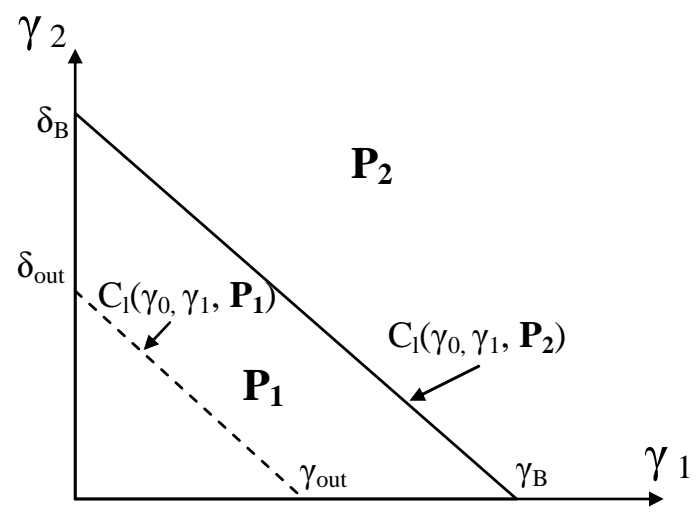

Fig. 4. Structure of power control regions for a fixed $\gamma_{0}$ and 2 subregions, using large power approximation. Regions $\mathcal{R}_{1}$ and $\mathcal{R}_{2}$ are separated by a line, $C_{l}\left(\gamma_{0}, \gamma_{1}, \mathbf{P}_{\mathbf{2}}\right)$. Below the dotted line $C_{l}\left(\gamma_{0}, \gamma_{1}, \mathbf{P}_{\mathbf{1}}\right)$, the power required to invert the channel is greater than $\mathbf{P}_{1}$, so the area below the dotted curve defines the outage probability.

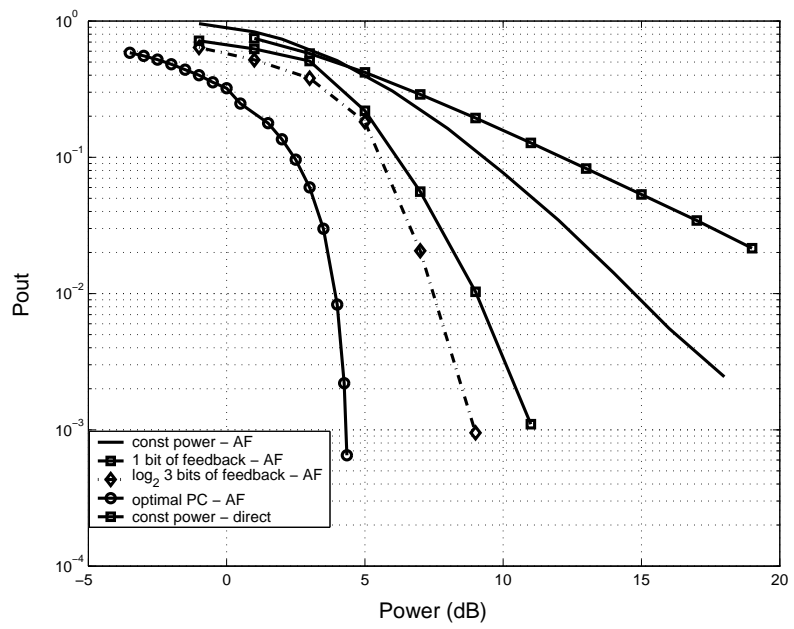

Fig. 5. Effect of more feedback bits on outage performance, for $\mathrm{d}=0.5, \alpha=3, \mathrm{R}=1 \mathrm{nats} / \mathrm{sec} / \mathrm{Hz}$ using the $\mathrm{AF}$ protocol. The relay in this case transmits with variable power in each time slot, and $P_{s}=P_{r}$. For comparison, the case of constant power transmission is shown, and also the optimal power control policy when perfect CSIT is available. Additionally the performance of a direct transmission system using constant power is shown. 


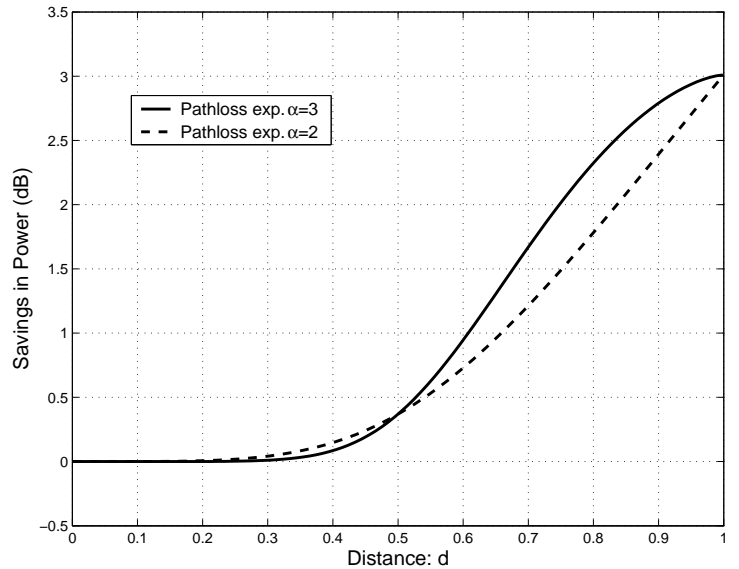

Fig. 6. Savings in power by using the optimal source-relay power ratio vs. equal power among source and relay assuming a rate $\mathrm{R}=1$ and $P_{\text {out }}=10^{-2}$. The $d$-axis represents the relay's fractional distance between the source and destination. The savings in power corresponds to the reduction in average power that is achieved by using the optimal power ratio versus equal power allocation between the source and relay. 